\title{
Ontology-based Collaborative Inter-organizational Knowledge Management Network
}

\author{
Nelson K. Y. Leung \\ RMIT International University \\ Vietnam, Ho Chi Minh City, Vietnam
}

nelson.leung@rmit.edu.vn

\author{
Seung Hwan Kang \\ Payap University, \\ Chiang Mai, Thailand
}

seung h@payap.ac.th

\author{
Sim Kim Lau and Joshua Fan \\ University of Wollongong, NSW, Australia
}

simlau@uow.edu.au; joshua@uow.edu.au

\begin{abstract}
Knowledge management encourages organizations to create and use knowledge continuously to gain competitive advantage. Some knowledge management approaches are industry specific, theoretical and procedure-wise without consideration of system interoperation. The lack of interoperability means heterogeneous systems from different organizations are unable to communicate, cooperate, exchange, and reuse knowledge with one another. Thus a collaborative interorganizational network is necessary. In this paper, we propose the use of ontology for organizations to access and retrieve inter-organizational knowledge in a similar domain. The incorporation of ontology and its mediation methods in the network allows organizations to reuse the interorganizational knowledge to support individual organizational knowledge management processes. A case study illustrating ontology-based collaborative inter-organizational knowledge management network over publication services among universities and business sectors is included.
\end{abstract}

Keywords: knowledge management, ontology, mapping, matching, merging, integration, interorganizational knowledge

\section{Introduction}

The reconcilability of ontology mediation allows the participant organizations to reuse interorganizational knowledge within the network even if there are fundamental differences in terms of structures and knowledge formats in their knowledge management systems (KMS). Under mutual agreement, organizations are permitted to retrieve inter-organization knowledge and the re-

Material published as part of this publication, either on-line or in print, is copyrighted by the Informing Science Institute. Permission to make digital or paper copy of part or all of these works for personal or classroom use is granted without fee provided that the copies are not made or distributed for profit or commercial advantage AND that copies 1) bear this notice in full and 2) give the full citation on the first page. It is permissible to abstract these works so long as credit is given. To copy in all other cases or to republish or to post on a server or to redistribute to lists requires specific permission and payment of a fee. Contact Publisher@,InformingScience.org to request redistribution permission. trieved knowledge can be reused to support the knowledge management (KM) processes, which include create, store, disseminate, use, and evaluate knowledge. Conventionally, technology has a very limited contribution in the knowledge creating stage, especially in socialization, externalization, and internalization phases and especially where tacit knowledge is involved. For example, word processing tools can be used 
to record and visualise explicit knowledge in externalization and internalization processes whereas communication tools such as email and telephone provide a platform for the exchange of explicit knowledge in socialization.

An ontology merging tool can provide a practical way to create knowledge by combining two or more ontologies semi- or fully-automatically in the network. This can be achieved on both a network and an organizational level. On the first level, the merging tool is capable of creating a shared ontology for a top-level mapping approach that contains common views of all organizational ontologies in the network. On the second level, an organization can create its own domain specific ontology by merging relevant ontologies from other organizations within the network. Other than that, an ontology integration tool provides an alternative way to create knowledge. Using an integration method, an organization can create its own knowledge by integrating relevant parts of ontologies from other organizations in the network into its own ontology building process. As a result, both merging and integration tools enable organizations to reuse, not only the contents of other ontologies, but also their associated inter-organizational knowledge stored in knowledge bases of other organizations. While ontology merging and integration are never a trivial task, even with the assistance of automatic tools, they are still less demanding than building from scratch.

A knowledge dissemination tool allows user to retrieve and use knowledge from an organizational knowledge repository. If a user cannot find suitable organization knowledge, $\mathrm{s} / \mathrm{he}$ has to seek it from other external sources. This can be achieved by creating mappings among ontologies of different organizations either semi- or fully-automatically with the support of an ontology mapping tool. The established mappings allow one KMS to access KMSs of other organizations in the same network when searching for knowledge. Besides, it is also practical for mapping to be performed on-the-fly. In this case, the automatic mapping tool is responsible to look for, select, and establish mapping with the most relevant concepts and properties from the other ontology in the network. Whenever the required knowledge is not available in the organizational repository, the KMS is able to retrieve and deliver inter-organizational knowledge in a "black box" through the establishment of mappings. In addition, inter-organizational knowledge can be reused to support the knowledge evaluation process. This is accomplished by setting up dedicated mappings between two or more ontologies. Once a piece of inter-organizational knowledge is updated, this inter-organizational knowledge will be translated into a suitable format and delivered from the source knowledge base to the target knowledge base automatically via pre-established mappings. To demonstrate the reconcilability of ontology mediation and reusability of inter-organizational knowledge in the network, this research investigates the use of an ontology and its related mediation methods which possess knowledge reusability and mismatch reconcilability to fill the gap.

This paper is organized as follows. The next section presents ontology in knowledge management. The third section includes a detailed review of various ontology mediation methods. We investigate the application of ontology and its related mediation methods that provide mechanisms of reconciling inter-organizational knowledge in the development of the proposed collaborative inter-organizational KM network. The case study section includes knowledge domain, analysis, design, and implementation of the reconciled inter-organizational knowledge in supporting the organizational KM process. The final section concludes the paper.

\section{Ontology Knowledge Management}

KM encourages organizations to create and use knowledge continuously for innovation and enhancement of services, products, and operations. Simultaneously, it also aims to improve the quality, content, value, and transferability of individual and group knowledge within an organization (Mentzas, Aposolou, Young, \& Abecker, 2001). The adoption of advanced data communication technology is important to enable an organization to access useful knowledge from anywhere 
in the network. However, some of the KM approaches range from industrial specific, theoretical, to procedure-wise, for example, the re-distributed KM framework is developed to manage organizational help desk knowledge (Leung \& Lau, 2006). Another example is the integrative framework that establishes an effective knowledge transfer process within an organization (Goh, 2002). These designs are incapable of cooperating in the current distributed knowledge environment, particularly areas that deal with organizational knowledge. Generally the approaches are customized to suit individual organizational KM strategies and business requirements without consideration of system interoperability. The lack of interoperability means heterogeneous KMSs from different organizations are not able to communicate, cooperate, exchange, and reuse knowledge with one another. In this case, the non-collaborative KMSs have several disadvantages for both knowledge workers and knowledge engineers. In the case of knowledge workers, they have to spend a lot of time and effort to look for relevant knowledge from different sources of KMSs in order to complete their works in the knowledge explosion era. For instance, an investment manager has to retrieve a company's financial reports, share performance reports, and retrieve regional economy reports from external sources if $\mathrm{s} / \mathrm{he}$ wants to make adjustment to the investment portfolio. In terms of knowledge engineers, they have to spend a lot of resources in creating and updating organizational knowledge although the same knowledge is available in other KMSs. As external sources of knowledge are essential for organizational performance, a new inter-organizational $\mathrm{KM}$ practice is required to enhance the interoperability among independent KMSs and to encourage the sharing of knowledge that crosses organizational boundaries in their business networks (Oinas-Kukkonen, 2005).

To improve inter-organizational KM practice, the use of an ontology is becoming increasingly important in the area of KM research. The concept of an ontology can also be applied to solve the interoperation problem in the distributed KMS environment. Ontology is defined as an explicit specification of a conceptualization, while a conceptualization is an abstract, simplified view of the world that we wish to represent for some purpose (Gruber, 1993).

In this approach, explicit knowledge of the KMS is annotated in a form that is machineprocessable metadata based on the domain or topic-specific ontology (Davies et al., 2005; Mentzas et al., 2001). Using the ontology, one KMS can communicate with others in spite of the underlying system, syntax, and structure heterogeneities, thus allowing the involved systems to understand incoming requests and return the required knowledge as they are using the same set of vocabularies. Besides, the exploitation of ontological metadata enables ontology-based searching to take place for the retrieval of a more precise collection of knowledge. For example, when a user is searching for banking knowledge, the user is required to search for "bank" as a concept of "financial institution", rather than "bank" as in the "river bank". This way the search engine is able to return relevant pieces of knowledge by examining the ontological metadata which has previously been added to the content of each piece of knowledge (Hasse, Volker, \& Sure, 2005).

Another way of adopting the ontological metadata in knowledge retrieval is by ontology browsing. For example, in the study of the help desk self-help KMS developed by Leung (2007), the ontologies contain the classification of technical problems and their related symptoms. Each instance in the ontology is linked with related resolutions stored in the knowledge base. To retrieve the resolution for a specific problem, the user is required to browse the ontologies and select the most appropriate instance. Another form of searching technique includes contextual searching (Hasse et al., 2005), which queries the domain (or topic) specific ontology for the retrieval of knowledge that is relevant to the user's current searching pattern.

\section{Ontology Mediation}

It is impractical to have the expectation that every individual and organization will agree on a common shared ontology (de Bruijn et al., 2006). Furthermore the adoption of such an approach 
is problematic. On one hand, it is a lengthy and non-trivial process to define and maintain a large globally shared ontology; on the other hand, the globally shared ontology approach may hinder a system from reflecting its actual business requirements due to the fact that design of the system is restricted by terminologies defined in the ontology (Visser \& Cui, 1998). Researchers such as Berners-Lee, Hendler, \& Lassila (2001) envision there will be a large number of small domain specific ontologies developed by communities, organizations, departments, or even individuals. With multiple ontologies available, individual systems could be designed based on its actual requirements without committing to a particular set of terminologies and heterogeneity (Visser, Jones, Bench-Capon, \& Shave, 1998). In addition, they are bound to have ontologies with inconsistent vocabularies and relationships; thus it is difficult for one system to understand and reuse other ontologies unless the ontologies can be reconciled in some form. This inconsistency problem associated with multiple ontologies is commonly termed as an ontology mismatch.

Based on the individual requirements, organizations and individuals will develop their own ontologies using different languages, scopes, coverage and granularities, modelling styles, terminologies, concepts, and encodings. To reuse other ontologies of different types, ontology mediation is required to reconcile the mismatches among heterogeneous ontologies in order for knowledge sharing and reuse (Predoiu et al., 2006). There are three major kinds of ontology mediations; these include mapping, merging, and integration. Ontology mapping is a process of relating similar concepts and relations from different ontologies to each other in which correspondences between different entities of the two ontologies are formulated as axioms in specific mapping language (de Bruijn et al., 2006; Klein, 2001). Since the involved ontologies do not require any adaptation, ontology mapping often specifies just a part of the overlap between ontologies that is relevant for the mapping application (Scharffe, de Bruijn, \& Foxvog, 2006).

Unlike mapping that links two separate ontologies together in a consistent and coherent form, ontology merging creates a new ontology by unifying two or more different ontologies, and it is usually hard to identify regions of the source ontologies from the merged ontologies (Pinto \& Martins, 2001). Compare with mapping that keeps the original ontologies unchanged, merging requires at least one of the original ontologies to be adapted so that the conceptualization and the vocabulary match in the overlapping parts of the ontologies (Ding, Fensel, Klein, \& Omelayenko, 2002). While a majority of the semantic web researchers foresee the main stream will switch to the approach of developing an enormous amount of small domain specific ontologies, McGuinness, Fikes, Rice, \& Widler (2000) argue that some industries and organizations still need to develop a very large and standardized ontology, such as SNOMED CT. SNOMED CT is a comprehensive clinical ontology developed by the College of American Pathologists, which contains about 344,549 distinct concepts and 913,697 descriptions (Lussier \& $\mathrm{Li}, 2004$ ). Theoretically, it is more efficient and effective to merge existing ontologies than to build a large ontology from scratch. In practice, the process of ontology merging is more than just simple revisions, improvements, or variations of the source ontologies (Lambrix, Habbouche, \& Perez, 2003; Pinto \& Martins, 2001). McGuinness et al. (2000) specify that the three major tasks that are required to merge two ontologies are: 1) coalesce two semantically identical terms from different ontologies so that they can be referred to by the same name in the resulting ontology; 2) identify terms that should be related by subsumption, disjointness, or instance relationships; 3 ) verify and validate correctness and consistency of the merged ontology. Chimaera, developed by the Stanford University Knowledge Systems Laboratory, is an example of a semi-automatic merging tool that supports the above three tasks.

Finally, the third type of ontology mediation is integration. Pinto and Martins $(2000,2001)$ define ontology integration as a process of building an ontology in one subject by reusing one or more ontologies in different subjects, and it is always possible to identify regions of the source ontologies from the integrated ontologies. Source ontologies may need some sort of refinements before 
they can be aggregated, combined, and assembled to form the resultant ontology. It is also important to include ontology integration in the early stage of the ontology building process, preferable during conceptualization and formalization in order to simplify the overall ontology building procedure.

\section{Case Study: Ontology-based Collaborative Inter- Organizational Knowledge Management Network}

\section{Knowledge Domain}

As mentioned in the previous section, knowledge created from external sources plays a very important role in supporting organizational activities because employees are often required to make use of the knowledge in their daily work. However, the majority of KM frameworks, KM practices, and KMSs are designed to handle or manage only organizational knowledge. Let us consider an example in a university. If a student is searching for information systems (IS) related literature (for instance, literature in the area of "inter-organizational KM"), the first thing s/he can do is to access the website of the university library. S/he can then enter a set of keywords in the search interface of the website to see whether the relevant literature is available in the library's collection, either in the form of hardcopy or softcopy. If so, s/he can choose to pick up the literature from the library or download in virtual format. Otherwise, s/he has to search again in various literature knowledge bases subscribed by the library. Literature knowledge bases allow subscribers to retrieve literature that includes journals, conference papers, electronic books, and theses in the forms of full text or abstracts and citations from their online knowledge repositories. Unfortunately, s/he has to search in every single knowledge base until s/he can find the required literature because each knowledge base contains different sets of literature based on publishers, disciplines, and so on. For instance, IEEE Xplore (IEEE) and ACM Digital Library (ACM) mainly contain computer related journal and conference papers that are published by IEEE and ACM respectively whereas Australian Digital Theses Program stores the theses of any disciplines that are produced by the higher research students in Australian universities. Finally, if s/he still cannot find any related literature, s/he may choose to search again using web search engines such as Yahoo and Google.

In this research, we propose to use an ontology and its related mediation methods. The ontology is incorporated to allow explicit knowledge to be annotated in the form of machine processable metadata. Although different organizations possess their own set of ontologies, the mediation methods are capable of reconciling the underlying heterogeneous ontologies. Each network contains knowledge of a specific domain. For example, an IS network should only provide knowledge in the domain of IS. Once an organization recognizes the need for a particular type of knowledge, the organization can invite other organizations that have the knowledge of a similar domain to establish a network together. When this network of knowledge matures, other organizations that want to use the knowledge may choose to join the network, instead of establishing their own individual knowledge network. Within the network, each organization commits to a mutual agreement to allow other participants to access an agreed section of the ontology and the associated knowledge repository in its knowledge base. In addition, an organization can commit to more than one network of different domains. For instance, library A may decide to commit to networks of IS, economics, mechanical engineering, education, and chemistry.

\section{The Proposed Ontology Mediation Method}

Before we continue to describe the proposed network, we want to point out that the participant organization first needs to make four important decisions related to ontology mediation. Figure 1 
shows a way to select the ontology mediation method. The first decision is to decide whether to adopt a top-level ontology or a one-to-one method as the network-level mapping approach. As this decision is based on the network level, the organizations as a whole may need to negotiate and compromise in order to select the most appropriate mapping approach for the benefit of the entire network. The decision process should include a thorough assessment of the aspects of resources, expertise, and frequency of modification. The top-level ontology approach can only be applied to an environment where the maintenance effort is minimal. Whenever a modification is performed in one of the ontologies in the network, the shared ontology at the top-level ontology approach may require a complete reconstruction. The organizations must also ensure that they have sufficient resources and expertise to build the shared ontology; otherwise, the one-to-one approach should be selected.

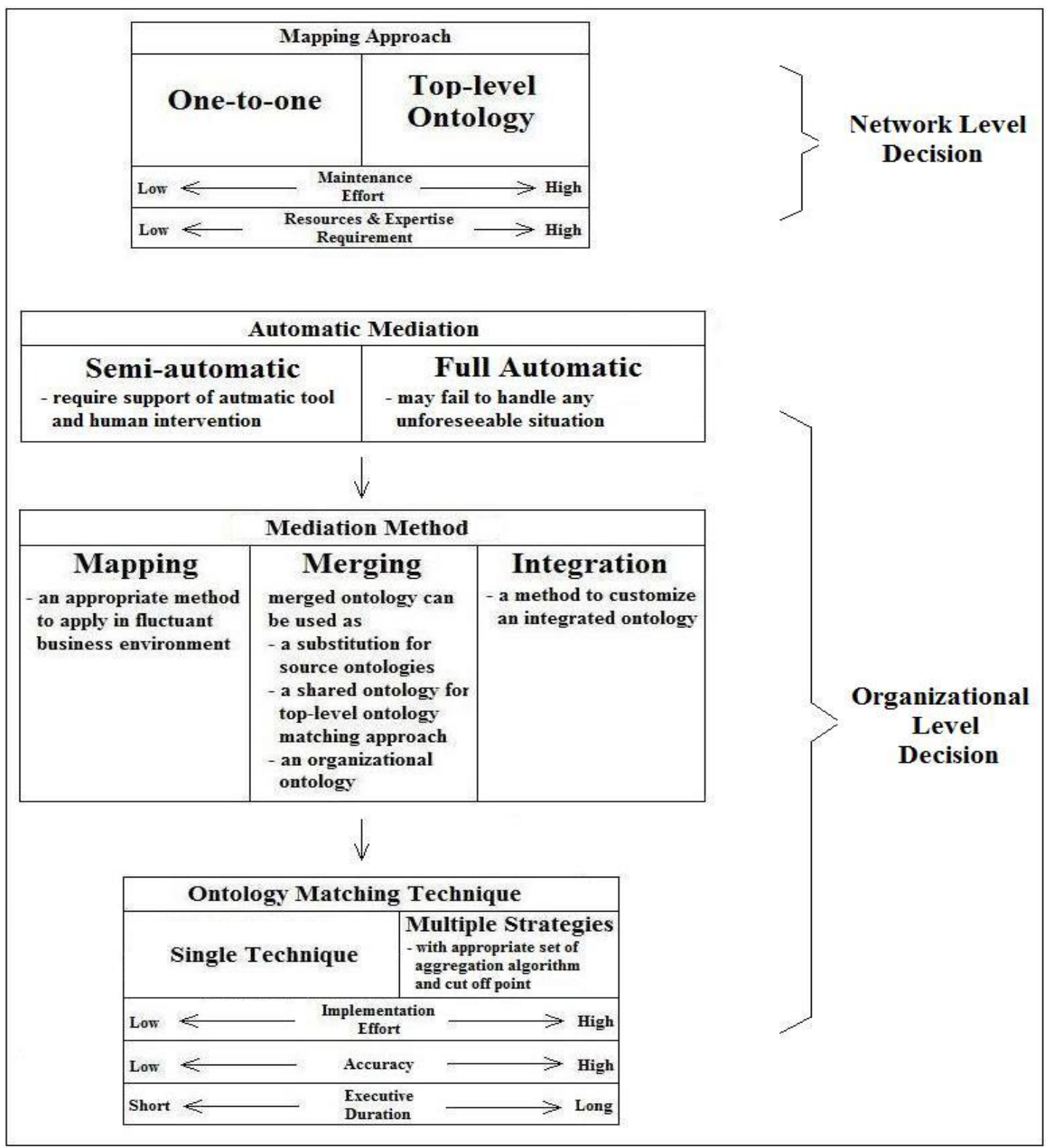

Figure 1: Selection matrix for ontology mediation

The second decision is to determine whether to perform mediation automatically or semiautomatically. Mediation can be performed semi-automatically, which requires the support of 
automatic tools as well as human intervention. Examples of support that can be provided by automatic tools include post-mediation verification, validation, and critiquing, as well as conflict recognition and resolution. Although semi-automatic mediation may have a better performance than the manual approach in terms of accuracy, it still relies substantially on human efforts and can be time consuming. As semi-automatic tool is not capable of supporting mediation on-the-fly, it would be ideal to perform mediation automatically. Unfortunately automatic tools are unable to detect and interpret concepts that do not have a close correlation, and it may also fail to handle any unforeseeable situations as the tool is designed to perform mediation under specific predefined condition.

The third decision is to decide whether to adopt merging, mapping, or integration as the preferred mediation method for each organization. Each organization can choose one or more methods based on its own need. The concept of mapping enables ontology to be developed in response to the organization's actual business requirement and is more appropriate in a dynamic business environment where ontologies are required to be modified frequently. Generally, no major reconstruction is required if an ontology has undergone a minor modification; for example, an ontology may only need to update its mappings accordingly if concepts are deleted from it. Alternatively, merging is an appropriate method for creating an ontology that combines common views of multiple-sourced ontologies. The merged ontology could act as: 1) a single ontology used to substitute individual source ontology; 2) a shared ontology used in the top-level ontology mapping approach; or 3) an organizational ontology that includes all possible views of other organizations' ontologies. Unlike merging, integration selects only appropriate modules from individualsourced ontologies to form an integrated ontology. Thus, integration is an appropriate method for organizations to construct tailored ontologies based on individual needs.

The final consideration is to decide whether to adopt single or multiple matching techniques. Organizations must take into consideration the execution duration, the level of acceptable matching accuracy, and the level of resources for implementation. In general, multiple strategies are expected to generate more accurate result than a single matching technique. The choice of aggregation algorithm and cut-off point also plays an important role in determining the level of matching accuracy. When choosing multiple strategies as its matching technique, the organization must conduct a series of experiments with the purpose of finding a combination of multiple strategies, aggregation algorithm, and cut-off point in order to produce the most accurate result. Compared to single matching technique, multiple strategies are relatively difficult to design and implement and also require longer execution time.

\section{Analysis}

Assume University A realizes that there is an increasing demand for IS related knowledge, however, this demand cannot be satisfied with the current collection of publications in the library. Consequently, University A decides to invite knowledge providers and libraries of other organizations to establish a network that contains IS related literature. Libraries from University B, University C and University D as well as Publisher ABC and Publisher XYZ agree to join. Except for University D, all other participating organizations possess ontologies. Figure 2a shows a partial view of the classification ontology adopted in the library of University A. In this ontology, the publication concept has concepts that include book, journal, proceeding, and thesis as its subclasses and each subclass is described by a set of properties such as International Standard Book Number (ISBN), International Standard Serial Number (ISSN), and publisher. The concept category and its subclasses are used to distinguish publications into different subjects, such as concepts of computer, medical, commerce, and computer science. Given that this network only provides knowledge in the domain of IS, the library of University A is willing to share publications that belong to the concept computer and its subclass information systems. As a publication may 
contain chapters written by different authors, the ontology reflects this by including concepts of book chapter, journal paper as well as conference paper and their related properties as an extension of concept book, journal, and proceeding respectively. Figure $2 b$ shows a partial view of the classification ontology in Publisher XYZ. There are three major concepts in this ontology, that is, concept book, journal, and proceeding. Each concept has a set of publication details (such as issue and edition), contains a set of literatures, and belongs to one discipline (such as information systems). The above three components are represented by concept publication details, literature, and discipline respectively. Similar to University A, Publisher XYZ has also agreed to share literature that is classified under the concept information systems.

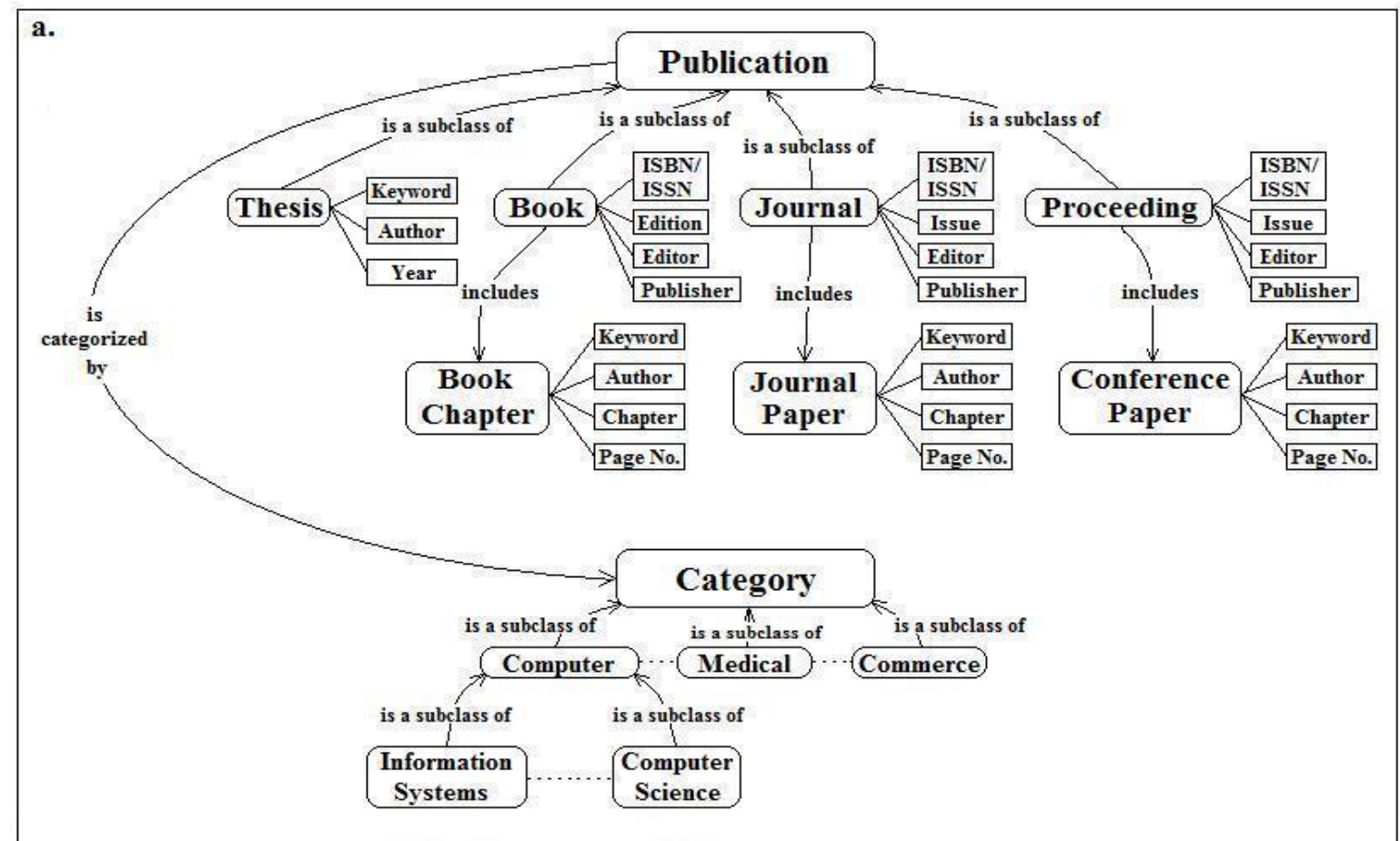

b.

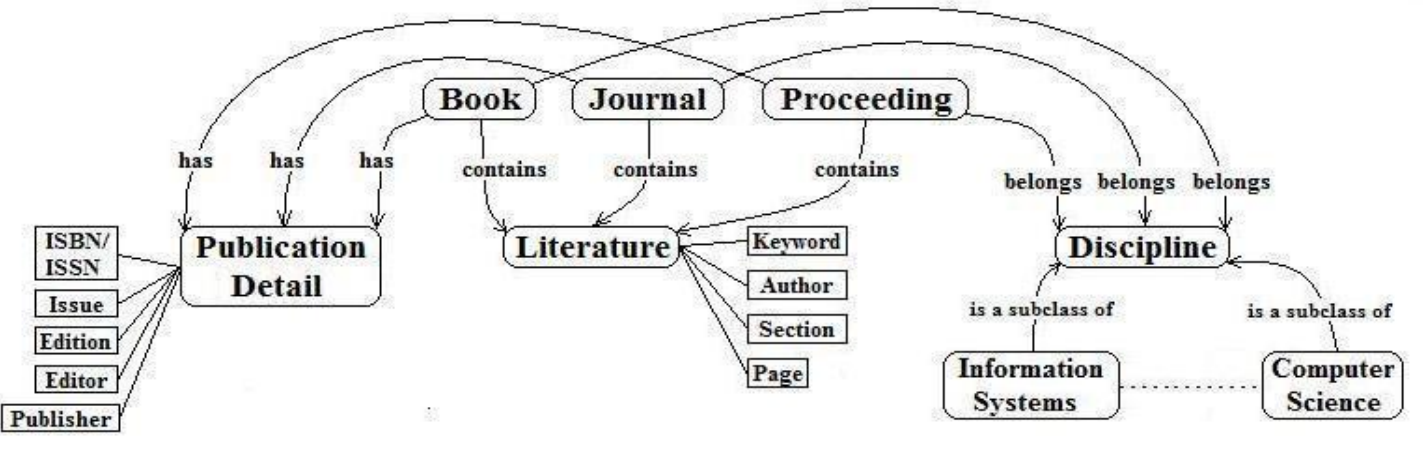

Figure 2: Partial view of the classification ontology adopted in the (a) Library of University $A$ and (b) Publisher XYZ.

Round Rectangular Nodes Represent Concepts. Rectangular Nodes and Labels on the Arcs Represent Properties.

\section{Design}

After careful consideration, the six organizations have reached a mutual agreement not to adopt top-level ontology as the network-wide mapping approach. This decision is based on the fact that 
there will be other organizations that will join the newly established network, so the shared ontology built for the top-level ontology mapping approach may require undergoing a series of reconstructions. At this moment, the organizations prefer to use the one-to-one mapping approach but they agree to review the mapping approach once the network becomes stable. Although they have sufficient expertise and resources to build and reconstruct the shared ontology, it is not cost effective to do so. In addition, the reconstruction works will affect the stability and performance of network-wide mediation because the shared ontology will be mapped by all other ontologies as a reference point.

As the library of University D does not possess an ontology, the library has to create one in order to fulfill the requirement of joining the network. Instead of building the ontology from scratch, the library decides to reuse ontologies from other organizations and integrate them into its own development process using the ontology integration method. However, the chosen ontologies must be similar to the library's actual classification in terms of publication and discipline in order to minimize the degree of modification. For instance, the concept publication and its subclasses in the ontology of University A are more appropriate than those defined in Publisher XYZ as the subclass thesis; book, journal and proceeding defined in the ontology of University A are very similar to the actual classification used in the library of University D. Thus the library reuses only a portion of the two ontologies that include the concept publication and its subclasses derived from the ontology of the University A as well as the concept discipline and its subclasses derived from the ontology of Publisher XYZ (see Figure 3).

In the ontology development process, the library of University D can reuse not only the ontologies of other organizations, it can also do so for the inter-organizational knowledge associated with the instance of the integrated ontology. As illustrated in Figure 3, the softcopy of the thesis described by the instance of the integrated ontology, thesis "Turning User into First Level Support in Help Desk: Development of a Web-based User Self-help KM System" in discipline IS, can be captured from the knowledge base of the University A and stored in the knowledge base of University D. This integrated ontology created by the library of the University D has resulted in additional function. By establishing dedicated mappings between the integrated ontology and its ontology providers (that is, the ontologies of University A and Publisher XYZ), the associated publication captured in the knowledge base of University D can be automatically updated as long as there is a revised version generated from the ontology providers. In this case, when the thesis "Turning User into First Level Support in Help Desk: Development of a Web-based User Selfhelp KM System" has undergone a minor revision in the knowledge evaluation process, the revised thesis will not only be stored in the knowledge base of University A, it will broadcast to other KMS through the dedicated mappings that includes the knowledge base of University D. 


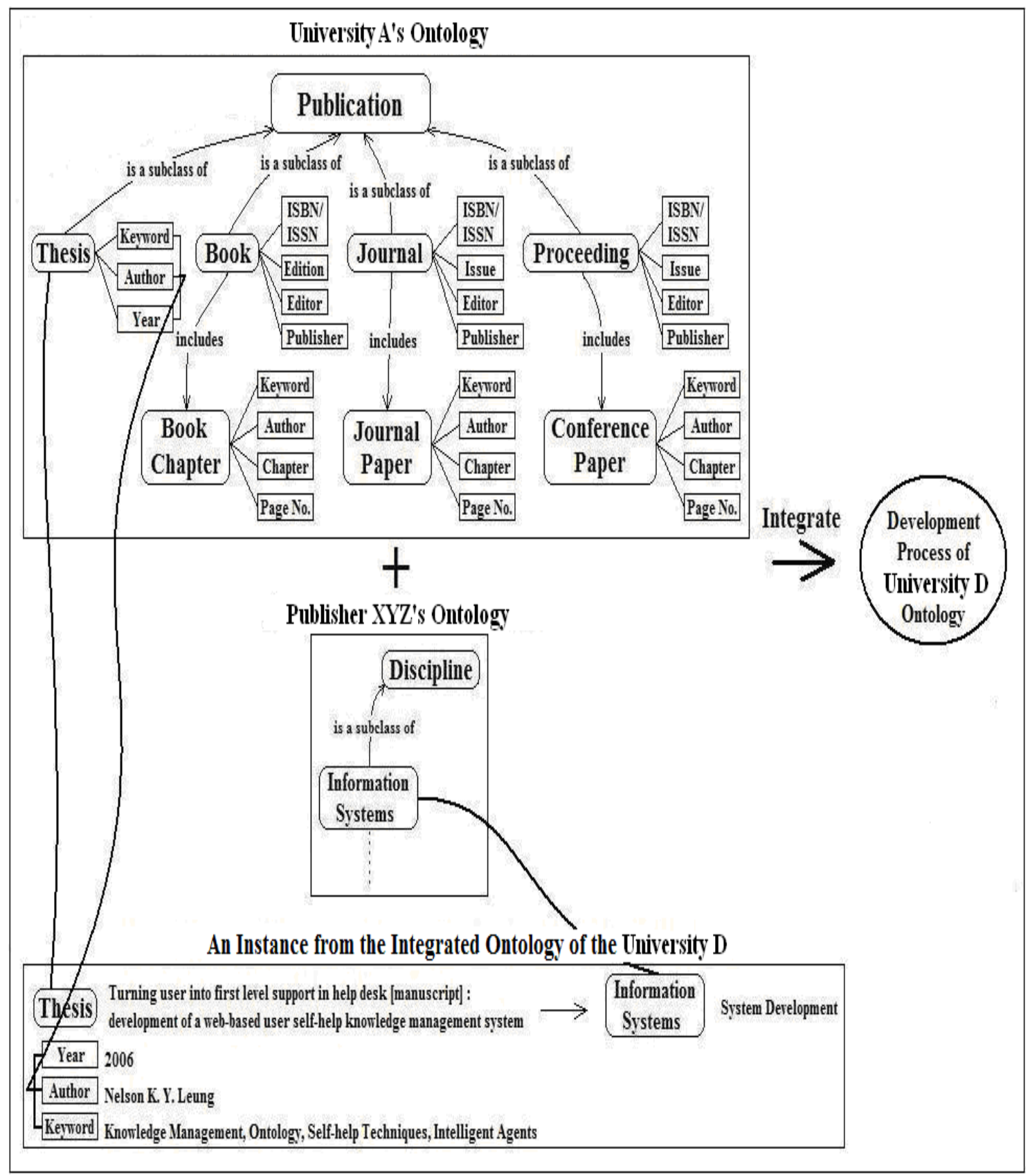

Figure 3: Process to develop university D's ontology using integration method

\section{Implementation}

To allow the general user to retrieve inter-organizational knowledge, an organization is required to establish mappings between its own ontology and ontologies of other organizations in this network. As shown in Figure 4, each broken line represents a mapping between a pair of concepts or properties that belong to two different ontologies. Making use of string-based and linguistic resources matching techniques, two similar concepts from the ontologies of $\mathrm{A}$ and $\mathrm{XYZ}$ are mapped with each other, for instance, two identical concepts (such as journal) and two properties that are synonyms (such as section and chapter) are mapped together. The mapping details of the two ontologies are summarized in Table 1. 


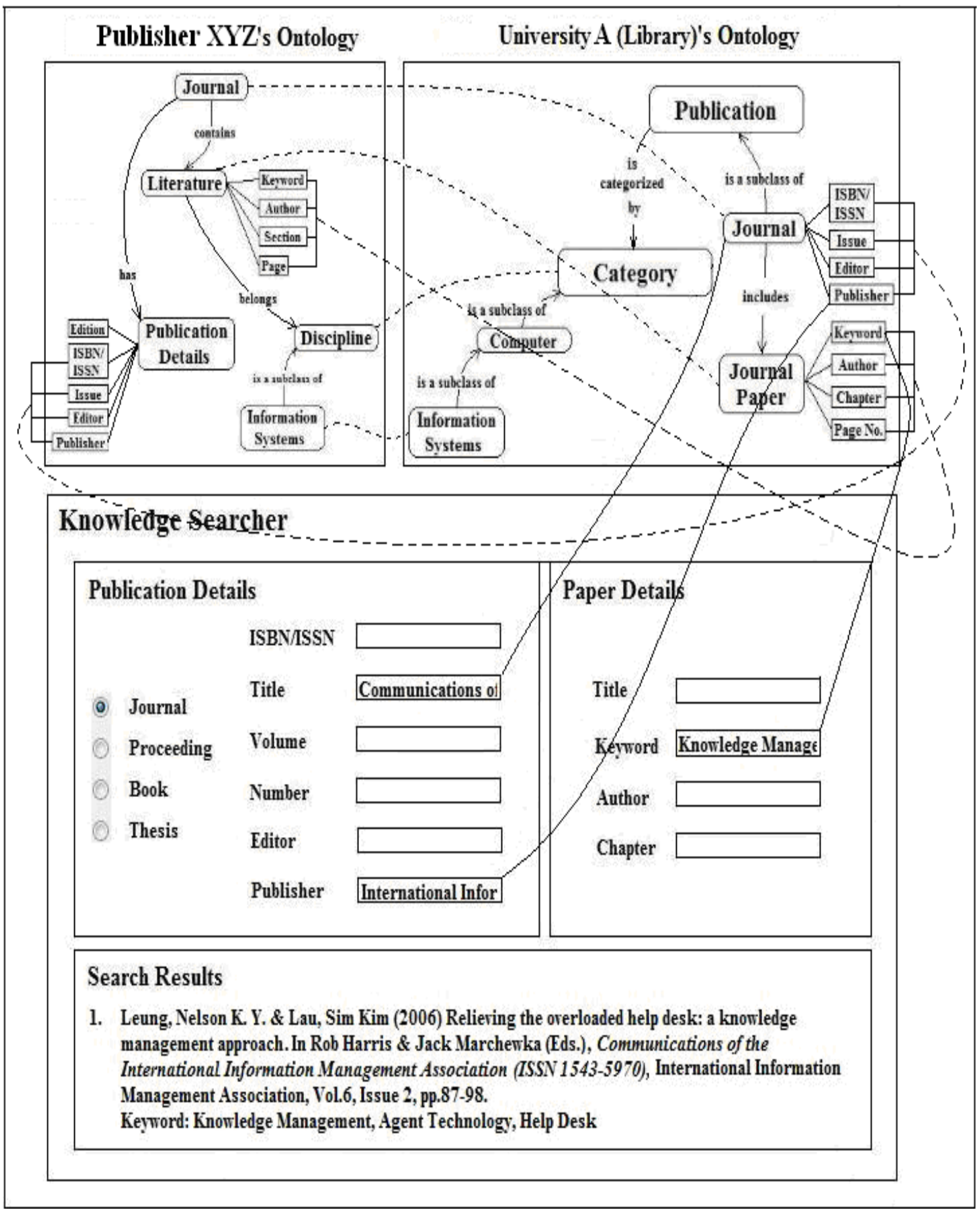

Figure 4: Inter-organizational knowledge retrieval and reusing process

In Figure 4, a user is searching for suitable journal papers by filling in data on title, publisher, and keyword fields on the "knowledge searcher" which is designed to be used as a search interface for the KMS at the library of University A. Since the KMS cannot provide a journal that satisfies this query, the system begins to search other KMS including Publisher XYZ. The mappings allow the KMS of Publisher XYZ to understand the incoming query. For example, the details provided in the title, publisher , and keyword fields on the search interface are similarly referring to the concepts journal, property publisher, and property keyword that belong to the ontology of Publisher 
$\mathrm{XYZ}$. As long as the requested journal is available in the knowledge base of Publisher XYZ, it will be delivered to the search interface of University A. Subsequently, the journal will be displayed as if it is retrieved from its own knowledge base. In other words, the entire interorganizational knowledge retrieval and displaying mechanism are performed in a "black box" manner.

Table 1: Mapping Summary of the Ontology of the Publisher XYZ and University A

\begin{tabular}{|l|l|l|l|}
\hline & XYZ Ontology & A Ontology & Matching Reason \\
\hline Concept & Journal & Joumal & Identical \\
\hline & Literature & Joumal Paper & Synonym \\
\hline & Discipline & Category & Synonym \\
\hline & Information Systems & Information Systems & Identical \\
\hline Property & ISBN/ISSN & ISBN/ISSN & Identical \\
\hline & Issue & Issue & Identical \\
\hline & Editor & Editor & Identical \\
\hline & Publisher & Publisher & Identical \\
\hline & Keyword & Keyword & Identical \\
\hline & Author & Author & Identical \\
\hline & Section & Chapter & Synonym \\
\hline & Page & Page No. & Identical \\
\hline
\end{tabular}

\section{Conclusion}

The organization based KM approaches have caused collaboration problem in which an organization is not capable of reusing inter-organizational knowledge even though the required knowledge is available in other organizations. An ontology-based collaborative inter-organizational KM network is proposed to solve the problems. To establish the network, a selection framework is proposed to assist organizations in selecting a suitable ontology mediation approach. The knowledge reusability and mismatches reconcilability of ontology and its related mediation methods enable organizational KMSs to understand an incoming request and the returned knowledge, thus making it possible for them to collaborate and communicate with each other. By annotating knowledge explicitly in the form of machine-processable representation, organizations joining the network can access, retrieve, and reuse domain specific inter-organizational knowledge to support the five stages of organizational KM process. While knowledge engineers can reuse interorganizational knowledge to create and evaluate organizational knowledge, general users benefit from the effectiveness and efficiency in searching for relevant inter-organizational knowledge.

\section{References}

Berners-Lee, T., Hendler, J., \& Lassila, O. (2001, May). The semantic web. Scientific American.

Davies, J., Duke, A., Kings, N., Mladenic, D., Bontcheva, K., Grcar, M., et al. (2005). Next generation knowledge access. Journal of Knowledge Management, 9(5), 64-84.

de Bruijn, J., Ehrig, M., Feier, C., Martin-Recuerda, F., Scharffe, F., \& Weiten, M. (2006). Ontology mediation, merging and alignment. In J. Davis, R. Studer, \& P. Warren (Eds.), Semantic web technologies. John Wiley \& Sons.

Ding, Y., Fensel, D., Klein, M., \& Omelayenko, B. (2002). The semantic web: Yet another hip? Data and Knowledge Engineering, 41(3), 205-227.

Goh, S. (2002). Managing effective knowledge transfer: An integrative framework and some practice implications. Journal of Knowledge Management, 6(1), 23-30.

Gruber, T. R. (1993). Toward principles for the design of ontologies used for knowledge sharing. International Journal of Human-Computer Studies, 43(5-6), 907-928. 
Hasse, P., Volker, J., \& Sure, Y. (2005). Management of dynamic knowledge. Journal of Knowledge Management, 9(5), 97-107.

Klein, M. (2001). Combining and relating ontologies: An analysis of problems and solutions. Proceedings of IJCAI-2001 Workshop on Ontologies and Information Sharing, 53-62.

Lambrix, P., Habbouche, M., \& Perez, M. (2003). Evaluation of ontology development tools for bioinformatics. BioInformatics, 19(12), 1564-1571.

Leung, N. K. Y. (2007). Easing the overloaded help desk: The development of an user self-help knowledge management system. VDM Verlag, Dr. Mueller.

Leung, N. K. Y., \& Lau, S. K. (2006). Relieving the overloaded help desk: A knowledge management approach. Communications of International Information Management Association (CIIMA), 6(2), 87-98.

Lussier, Y. A., \& Li, J. (2004). Terminological mapping for high throughput comparative biology of phenotypes. Proceedings of the Pacific Symposium on Biocomputing, 202-213.

McGuinness, D. L., Fikes, R., Rice, J., \& Widler, S. (2000). An environment for merging and testing large ontologies. KR2000: Principles of Knowledge Representation and Reasoning, 483-493.

Mentzas, G., Aposolou, D., Young, R., \& Abecker, A. (2001). Knowledge networking: A holistic solution for leveraging corporate knowledge. Journal of Knowledge Management, 5(1), 94-106.

Oinas-Kukkonen, H. (2005). Towards evaluating knowledge management through the 7C model. Proceedings of the European Conference on Information Technology Evaluation.

Pinto, H. S., \& Martins, J. P. (2000). Reusing ontologies. Proceedings of the AAAI Spring Symposium Series, Workshop on Bringing Knowledge to Business Processes, 77-84.

Pinto, H. S., \& Martins, J. P. (2001). A methodology for ontology integration. Proceedings of the 1st International Conference on Knowledge Capture, 131-138.

Predoiu, L., Feier, C., Scharffe, F., de Bruijn, J., Martin-Recuerda, F., Manov, D., et al. (2006). State-ofthe-art survey on ontology merging and aligning V2. EU-IST Integrated Project (IP) IST-2003-506826 SEKT: Semantically Enabled Knowledge Technologies, University of Innsbruck.

Scharffe, F., de Bruijn, J., \& Foxvog, D. (2006). Ontology mediation patterns library V2. EU-IST Integrated Project (IP) IST-2003-506826 SEKT: Semantically Enabled Knowledge Technologies. University of Innsbruck.

Visser, P. R. S., \& Cui, Z. (1998). On accepting heterogeneous ontologies in distributed architectures. Proceedings of the ECAI'98 workshop on Applications of Ontologies and Problem-solving methods, 112119.

Visser, P. R. S., Jones, D. M., Bench-Capon, T. J. M., \& Shave, M. J. R. (1998). Assessing heterogeneity by classifying ontology mismatches. Proceedings of the International Conference on Formal Ontology in Information Systems, 148-162.

\section{Biographies}

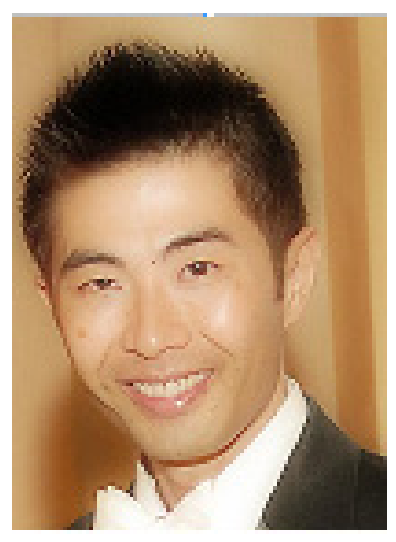

Mr. Nelson Leung has a Bachelor of Information Technology from the Queensland University of Technology, a Master of Information Systems from the Griffith University and a Master of Information Systems (Research) from the University of Wollongong. Nelson is now working as a full-time BIS Lecturer at the RMIT International University Vietnam. He is also a PhD candidate at the University of Wollongong and an Adjunct Researcher at the Payap University. Nelson has published widely in refereed book, journals and international conferences. Prior to that, he was an Instructor at the University of Wollongong. 
Dr. Seung Hwan Kang is a Lecturer in the Department of Computer Information Systems at the Payap University. His research primarily focuses on the Semantic Web and Ontology. His recent work is in the Semantic Web use cases that extend the current Web by providing well-defined meaning in a context of Accounting Information Systems (AIS), e-Business, e-Tourism, Knowledge Management and other research interests. His publications include papers in the IEEE, Springer and Inderscience.

Dr. Sim Kim Lau is a Senior Lecturer in the Information Systems Discipline, School of Information Systems and Technology at the University of Wollongong. She has published widely in refereed Information Systems journals and international conferences. Her research interest is in the area of application of artificial intelligence and optimization techniques to business problems, and more recently in the area of semantic web and ontology. Her teaching covers knowledgebased information systems and decision support systems.

Dr. Joshua Fan is a Lecturer at the Sydney Business School, teaching in the areas of logistics and supply chain management, project management, management information systems, operations and production management. Joshua has supervised a number of research students and he undertakes research collaborations with colleagues at Anderson Graduate School of Management, University of California, Riverside in the United States and Assumption University in Bangkok, Thailand. His research interests centre on operations research, supply chain management, electronic business and workflow optimizations. 